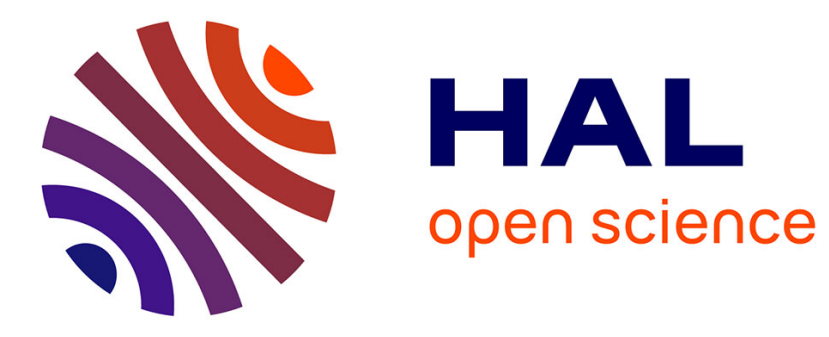

\title{
Optimization of mobile sensor coverage with UAVs
}

Christelle Caillouet, Frédéric Giroire, Tahiry Razafindralambo

\section{To cite this version:}

Christelle Caillouet, Frédéric Giroire, Tahiry Razafindralambo. Optimization of mobile sensor coverage with UAVs. 11th International Workshop on Wireless Sensor, Robot and UAV Networks (WiSARN@IEEE INFOCOM 2018), Apr 2018, Honolulu, United States. 10.1109/INFCOMW.2018.8406980 . hal-01850442

\section{HAL Id: hal-01850442 \\ https://hal.inria.fr/hal-01850442}

Submitted on 27 Jul 2018

HAL is a multi-disciplinary open access archive for the deposit and dissemination of scientific research documents, whether they are published or not. The documents may come from teaching and research institutions in France or abroad, or from public or private research centers.
L'archive ouverte pluridisciplinaire $\mathbf{H A L}$, est destinée au dépôt et à la diffusion de documents scientifiques de niveau recherche, publiés ou non, émanant des établissements d'enseignement et de recherche français ou étrangers, des laboratoires publics ou privés. 


\section{Optimization of mobile sensor coverage with UAVs}

\author{
Christelle Caillouet, Frédéric Giroire \\ Université Côte d'Azur, CNRS, Inria, I3S, France
}

\author{
Tahiry Razafindralambo \\ Université La Réunion, LIM, France
}

\begin{abstract}
In this work, we address the optimization problem of covering a set of mobile sensors with a fleet of flying devices. The goal is to deploy a connected set of Unmanned Aerial Vehicles (UAVs) continuously monitoring the mobile sensors and reporting information to a fixed base station for efficient data collection. We propose an effective optimization model reducing the number of variables of the problem and solved using column generation. Results show that our model is tractable for large topologies with several hundreds of possible 3D locations for the UAVs deployment and provides integer solutions with the generated columns very close to the optimum. Moreover, the deployment changes among time remains low in terms of number of UAVs and cost, to maintain connectivity and minimize the data collection delay to the base station.
\end{abstract}

\section{INTRODUCTION}

The use of wireless aerial devices is a promising approach to improve the performance of Wireless Sensor Networks (WSNs) that mainly focus on the observation of the physical world [1]. In particular, Unmanned Aerial Vehicles (UAVs) can act as flying base stations to enhance the coverage and performance of WSNs in different application domains such as civilian (environment) [2], [3], military (battlefield) [4] and industrial (structural monitoring) [5], [6]. WSNs make the network operations easier, cheaper and can increase data accuracy compared to cellular or fixed terrestrial networks. Easier because wireless sensor networks are configured to be autonomous. Cheaper due to the decreasing cost of electronics. Accurate due to the increasing number of deployed sensors and using network connections which can provide and spread results during time. A proper placement of wireless sensors over the area of interest is a critical job especially when mobility is involved. In recent applications involving tracking, sensors are moved over the area of interest in an unpredictable way and in remote locations that do not ensure directly wireless communications between the sensors. In such conditions data gathering is difficult. Deploying a set of Unmanned Aerial Vehicles (UAVs), or flying drones, is therefore an effective way to track and gather data from moving sensors.

Data gathering and coverage of mobile sensors by a set of UAVs is a complex task but allows a more flexible and efficient deployment. The UAVs can track and continuously report information from the observed sensors to a sink for analysis. Moreover, one UAV can cover multiple sensors depending on its altitude, which reduces the number of UAVs to be deployed. To minimize the deployment cost of a fleet of UAVs to cover a set of mobile sensors, multiple parameters should be considered including three-dimensional UAVs position, connectivity and cost. UAVs movement and placement have to be consistent regarding the ground sensors for coverage and data gathering. Each ground sensor must be covered by at least one UAV and the network of UAVs has to be connected to a fixed sink for the data gathering process [7]. Unlike sensors, deploying UAVs has a much higher cost (price, battery, ...). This deployment cost should be minimized.

The goal of this paper is to provide optimization-based models and frameworks determining bounds on the optimal UAVs deployment for mobile sensor coverage. Our work considers an exact model that can be solved optimally and that provides the minimum deployment cost for a full mobile sensor coverage. We ensure an effective data collection from the sensors to a base station and investigate the evolution of the UAVs location among time. To the best of our knowledge, we are the first to propose an exact model, which scales well with the number of sensors and 3D-locations of UAVs. To solve the coverage problem of mobile sensors using a fleet of UAVs, we use column generation that is an optimization technique preventing the enumeration of an exponential number of variables, or columns, to solve a linear program. We show that with our method we can obtain optimal solutions of the problem for more than two hundreds possible 3D locations for the UAVs.

The reminder of this paper is organized as follows. The next section introduces the related work. In Section III, we describe the network model used for sensors and flying drones. In Section IV, we present our optimization model involving special structure of connected sets of positions for the UAVs. We describe the column generation used as resolution method in Section IV-C. The optimization model is validated in Section V. Solutions show the effectiveness of the model that uses only a small number of variables associated with sets of possible deployment for the UAVs at each time. We also investigate the changes of aerial positions among time. Finally, we conclude the paper in Section VI.

\section{RELATED WORK}

Coverage by a fleet of UAVs or mobile devices have been studied by many different viewpoints but especially from two research communities. From the robotics point of view, the main objective is the collaboration among mobile devices for a specific task [8]. The robotic community focuses on the deployment itself or how to drive each device to its specific location while maintaining connectivity among the device while ensuring good coverage of the area to monitor [7]. From the ad hoc and sensor network community, given a location 
for each device, the objective is to implement protocols above the deployed network [9], [10].

Another way to tackle the issue is from the optimization point of view. Multiple works proposed mathematical formulations for the coverage and positioning problems using UAVs, to find optimal or approximated solutions [11], [12], [13], [14]. Several objective functions have been considered. In [11], the authors try to maximize the end-to-end throughput in a cellular network with UAVs. In [13], a single path of UAVs acting as communication relays is optimized. In [13], dynamic programming is used to find an optimal routing for two camera-equipped UAVs cooperatively tracking a single target moving on the ground. In [12] the authors focus on linear connected topology construction. In contrast of these works, we focus on a 3D model to deploy multiple UAVs that can monitor mobile sensors and provide a connected backbone to collect information to a central base station. An interesting point raised by the authors of [12] is related to the coverage aspect. They assume that coverage is an attenuated disc in comparison with our case, where we assume a complete and full coverage disc for the UAVs.

We extend previous results from the literature on the optimal coverage of ground sensors by a set of flying drones. In [15], the ground sensors are static, while in this paper, they are mobile and can move across the area of interest. In [16], authors do not ensure connectivity among the UAVs, and their primary focus is energy consumption. We have seen that ensuring UAV connectivity with a fixed base station is interesting to collect efficiently information from the mobile ground sensors to a central entity in order to be efficiently analyzed as studied in [7], [8]. In [5], authors consider maximizing the total coverage area of the UAVs and their lifetime. But in their model, all UAVs are assumed to be placed at the same altitude and are not connected with each other.

\section{THE MOBILE SENSOR COVERAGE PROBLEM WITH CONNECTED SETS OF UAVS}

Time is discretized so that a position of the sensors at each time is estimated and given by the successive sets $N^{t}$, for $t \in\{0, \ldots, T\}$, where $T$ is the observation time period length. The goal is to ensure that all the sensors are covered at all time with connected sets of flying drones. In order to minimize the deployment cost, we seek efficient sets of UAVs among time : We want to globally use the minimum number of flying drones at low altitude, closed to the base station, and minimize their moves across time.

At each time step, the constraints of our problem are :

(i) ensuring the coverage of the sensors depending on their current location;

(ii) ensuring connectivity among UAVs and a base station.

The sensors are placed on the ground and associated with two dimensional coordinates $\left(x_{n}, y_{n}\right)$ for each $n \in N^{t}$. Each UAV $u$ can be located in the three dimensional space. Let $P$ be the set of possible 3D locations to deploy the UAVs, and $p=\left(x_{u}, y_{u}, h_{u}\right) \in P$ be respectively the position $\left(x_{u}, y_{u}\right)$ of UAV $u$ in the 2D plane, and $h_{u}$ its altitude.
We derive the observation radius $r_{u}^{h}$ of UAV $u$ as a function of its altitude $h_{u}$ and of its directional antenna half beamwidth $\theta$, also called visibility angle of the UAV. The coverage area of UAV $u$ on the 2D plane, given the visibility angle $\theta$ and the altitude $h_{u}$, is represented by a disk of radius bounded by:

$$
r_{u}^{h} \leq h_{u} \cdot \tan \left(\frac{\theta}{2}\right) .
$$

We say that an UAV $u$ deployed at location $p \in P$ covers sensor $n \in N^{t}$ if the distance between its projection on the 2D plane and the sensor $d(u, n)=\sqrt{\left(x_{u}-x_{n}\right)^{2}+\left(y_{u}-y_{n}\right)^{2}}$ is below the observation radius $r_{u}^{h}$ of the UAV. Similarly, for air-to-air communications, an UAV $u$ can communicate with another UAV $v$ if their distance in the 3D space is such that $D_{u v}=\sqrt{\left(x_{u}-x_{v}\right)^{2}+\left(y_{u}-y_{v}\right)^{2}+\left(h_{u}-h_{v}\right)^{2}} \leq R_{u}$, where $R_{u}$ is the communication range of UAV $u$.

For efficient data collection, we enforce the deployed UAVs to be connected with each other and with a fixed base station $b$ located on the ground at coordinates $\left(x_{b}, y_{b}, 0\right)$.

The overall goal of our optimization problem is to minimize the deployment cost of the UAVs during the monitoring time period. In order to take into account several parameters related to this type of infrastructure and optimize the wireless communications, we define a particular cost representation in the next section. Indeed, we want to optimize the aerial data collection, not only the air-to-air communications by ensuring a connected backbone of UAVs with the base station and the delay of data gathering, but also the air-to-ground communications by trying to deploy the UAVs at low altitude.

\section{LINEAR PROGRAMMING FORMULATION}

In order to efficiently solve our problem, we present a new formulation of the mobile sensor coverage problem. The formulation is based on a decomposition model and is solved using column generation. This formulation allows us to separate our problem into two subparts: (i) a master problem in which we deal with time and ensure a complete coverage of the sensors at each time depending on their current locations, and (ii) a pricing problem generating subsets of UAVs connected with the base station and covering the sensors at a given time.

\section{A. Master program}

Variables of the linear program do not depend on one specific UAV location, but on more sophisticated structures, i.e. subsets of deployed UAVs with specific characteristics. Therefore, in the master problem, we do not deal with possible positions for each UAV, but only seek to select one subset of connected UAVs at each time in order to cover the sensors.

Definition 1 (Covering set). Let $S \subseteq P$ be a subset of $3 D$ positions with the following properties :

- One deployed UAV is associated with each 3D-position $\left(x_{p}, y_{p}, h_{p}\right) \in S$;

- Nodes of $S$ form a connected graph with the base station $b$, i.e. it exists a path between $b$ and every $u \in S$.

The goal is to find an UAV positioning at each time such that, (i) the UAV stays close to the base station for efficient 
data collection, (ii) the UAV is at the lowest altitude possible to ensure good connectivity with the sensors, and (iii) we use globally the minimum number of UAVs. In order to take into account the optimization of the UAVs positioning, we define the following metric associated with the subsets of UAVs.

Definition 2 (Deployment cost). For each subset of UAVs $S$, we compute an associated cost depending on the 3D-location of the UAVs:

$$
C_{S}=\sum_{u \in S} D_{b u}
$$

where $D_{b u}=\sqrt{\left(x_{u}-x_{b}\right)^{2}+\left(y_{u}-y_{b}\right)^{2}+\left(h_{u}\right)^{2}}$ is the distance on the 3D-plane between $U A V u \in S$ and the base station $b$.

Let $\mathcal{S}$ denote the set of all possible subsets $S . \mathcal{S}$ has an exponential size. The goal here is to prevent the enumeration of all possible subsets of UAVs using a known technique of optimization called column generation. We present the linear formulation of the master problem, and its associated pricing problem in the next section, before describing the resolution method in Section IV-C.

$$
\begin{array}{r}
\min \quad\left(\sum_{t=0}^{T} \sum_{S \in \mathcal{S}}\left(z_{S}^{t} \cdot C_{S}\right)+\right. \\
\left.\max _{t \in[1, T]}\left|\sum_{S \in \mathcal{S}}\left(z_{S}^{t} \cdot C_{S}\right)-\sum_{S^{\prime} \in \mathcal{S}}\left(z_{S^{\prime}}^{t-1} \cdot C_{S^{\prime}}\right)\right|\right) \\
\sum_{S \in \mathcal{S}} z_{S}^{t}=1, \forall t \in[0, T] \\
\chi_{n}^{t} \geq 1, \forall t \in[0, T], n \in N^{t} \\
\chi_{n}^{t} \leq \sum_{S \in \mathcal{S}}\left(z_{S}^{t} \cdot \sum_{u \in S}\left\lfloor\frac{r_{u}^{h}}{d_{u n}}\right\rfloor\right), \forall t \in[0, T], n \in N^{t} \\
z_{S}^{t}, \chi_{n}^{t} \in\{0,1\}
\end{array}
$$

The objective function (1) seeks to minimize the global deployment cost and the changes between consecutive subsets of UAVs among time. The metric used here is the one defined in Definition 2. The first part of the objective function ensures that we deploy the minimum number of UAVs as close to the base station as possible, ensuring the lowest altitude to cover the sensors and the fastest delay to gather the monitored information. In the second part of the objective function, we minimize the difference between the value of the cost for two consecutive time slots, meaning that the distances between the UAVs and the base station should remain the same among time. The reason is twofold. First, we want to keep a selected set as long as possible without any changes. In that case the UAVs remains stationary which is good for the air-to-air and air-to-ground communications. Second, if the set of UAVs is not valid anymore, then we want to keep the same number of them, or at the same distance of the base station. Indeed, adding/removing UAVs is very costly in terms of the drone itself, and of the wireless communication establishment when reaching/leaving its position.

To get rid off the absolute value, we introduce a new variable $\lambda \geq 0$. The objective function becomes :

$$
\min \left(\sum_{t=0}^{T} \sum_{S \in \mathcal{S}}\left(z_{S}^{t} \cdot C_{S}\right)+\lambda\right),
$$

and we add the following constraints to the program:

$$
\begin{aligned}
& \lambda \geq \sum_{S \in \mathcal{S}}\left(z_{S}^{t} \cdot C_{S}\right)-\sum_{S^{\prime} \in \mathcal{S}}\left(z_{S^{\prime}}^{t-1} \cdot C_{S^{\prime}}\right), \forall t \in[1, T] \\
& \lambda \geq \sum_{S \in \mathcal{S}}\left(z_{S}^{t-1} \cdot C_{S}\right)-\sum_{S^{\prime} \in \mathcal{S}}\left(z_{S^{\prime}}^{t} \cdot C_{S^{\prime}}\right), \forall t \in[1, T]
\end{aligned}
$$

Minimizing $\lambda$ thus gives the minimum absolute value of the cost evolution between two consecutive time slots.

The master problem has thus only 3 sets of constraints. Constraints (2) state that we must select exactly one subset $S$ of UAVs at each time $t$. Constraints (3) and (4) ensure that all the sensors are covered at each time if they are within the coverage area of at least one UAV of set $S$. It is worth noting that we must round down the fraction $\frac{r_{u}^{h}}{d_{u n}}$ since we should get 0 when the distance between UAV $u$ and sensor $n$ is greater than the coverage radius of $u$. Indeed, the sensor is outside the coverage area of the UAV, so it should not be covered. If we do not round down, then the value of the fraction is below 1 , but summing over all $u \in S$ could increase over 1 and then allow the value of variable $\chi_{n}^{t}$ to be equal to 1 which is not true. Rounding down ensures an exact coverage since the value of the sum in constraints (3) exceeds 1 only if, for at least one $u \in S$, the coverage radius of $u$ is greater than its distance with the sensor.

\section{B. Pricing program}

The sub-problem, or pricing problem, aims at generating subsets of deployed UAVs fulfilling the property of forming a connected backbone of UAVs with the base station. It is based on the dual formulation of the master problem.

Given the master problem (1)-(5), let $\beta^{(i)}$ be the dual variables associated with constraints $(i)$. For each $S \in \mathcal{S}, t \in$ $[0, T]$, the associated dual constraint is of the form:

$$
C_{S} \cdot(1-\gamma)-\sum_{n \in N^{t}} \beta_{n t}^{(4)} \sum_{u \in S}\left\lfloor\frac{r_{u}^{h}}{d_{u n}}\right\rfloor \geq \beta_{t}^{(2)}
$$

where $\gamma$ is a coefficient involving dual variables $\beta^{(6)}$ and $\beta^{(7)}$ depending on the value of $t$ (i.e. $\gamma=f\left(\beta^{(6)}, \beta^{(7)}\right)$ ).

Given the dual constraints associated with variables $z_{S}^{t}$ related with the subsets of UAVs and time, and the values of the dual variables $\beta^{(i)}$ as input of the pricing problem, we seek to compute minimum weighted connected subsets of UAVs violating these constraints to add to the set of columns of the master problem.

Thus, a minimum weighted connected subset generation either gives a good candidate to add to the set of variables of the master problem, or proves that no such column exists. If the cost computed by the pricing problem is smaller than $\beta^{(2)}$, the generated subset is added to the set of variables. Constraints of this problem define the structure of the subsets of UAVs. Given the set $P$ of possible 3D-locations for the 
UAVs, the goal is to select a subset of locations to deploy UAVs, such that they form a connected backbone with the base station in order to efficiently collect data from the mobile sensors on the ground.

In order to improve the efficiency of the computed subset, we define a pricing problem for every $t \in[0, T]$, and we specify that the connected subset must cover the sensors at that time $t$. To do so, we introduce variables $y_{p} \in\{0,1\}$ determining if location $p \in P$ is chosen to deploy an UAV included in the subset. The objective of the pricing program is to minimize the weighted connected subset cost depending on the dual constraint presented above. The constraints of the pricing problem are general flow constraints. We ensure the existence of a flow between the base station and the sensors $n \in N^{t}$ (Constraints (9)), and ensure that no flow can go through unchosen locations, or if the two UAVs are far away from each other (Constraints (10) and (11)). Similarly, if a location $p$ is not chosen or not covering a sensor $n \in N^{t}$, then no flow can go between $p$ and $n$ (Constraints (12)).

$$
\begin{aligned}
& \min \sum_{p \in P} y_{p}\left(C_{p}(1-\gamma)-\sum_{n \in N^{t}} \beta_{n t}^{(4)}\left\lfloor\frac{r_{p}^{h}}{d_{p n}}\right\rfloor\right) \\
& \sum_{q \in P, q \neq p} f_{p q}-\sum_{q \in P, q \neq p} f_{q p}= \begin{cases}\left|N^{t}\right| & \text { if } p=b \\
0 & \text { if } p \in P \\
-1 & \text { if } p \in N^{t}\end{cases} \\
& \forall p \in P \cup N^{t} \cup\{b\} \\
& f_{p q} \leq y_{p} \cdot\left\lfloor\frac{R_{p}}{D_{p q}}\right\rfloor \cdot\left|N^{t}\right|, \quad \forall p, q \in P \\
& f_{p q} \leq y_{q} \cdot\left\lfloor\frac{R_{p}}{D_{p q}}\right\rfloor \cdot\left|N^{t}\right|, \quad \forall p, q \in P \\
& f_{p n} \leq y_{p} \cdot\left\lfloor\frac{r_{p}^{h}}{d_{p n}}\right\rfloor \cdot\left|N^{t}\right|, \quad \forall p \in P, n \in N^{t} \\
& f_{p q} \in \mathbb{R}, y_{p} \in\{0,1\}
\end{aligned}
$$

\section{Resolution method}

Column generation is a decomposition method that combines the resolution of a restricted master problem, i.e. the master problem with a limited number of variables/columns allowing the existence of at least one feasible solution of the linear relaxation of the problem, and a pricing problem or subproblem generating new columns to add to the master problem in order to improve the objective value. When the pricing problem becomes unfeasible, then by the separation/optimization theorem we know that the optimal value of the relaxed master problem has been reached.

We use column generation to optimally solve the linear relaxation of the master problem with restricted set of initial variables. Indeed, we know that there exists an exponential number of subsets of UAVs. At the beginning of our resolution process, we only generate one subset $S_{0}$ containing all the possible 2D-locations for UAVs, and placing them at the highest altitude available at these locations. This set $S_{0}$ is actually the most covering set, ensuring an initial coverage of the sensors at each time. However, this solution is not efficient in terms of objective value corresponding to the deployment cost. Placing an UAV at every possible location is very costly, and assigning them the highest possible altitude degrades the air-to-ground communication quality. Consequently, the column generation process solves iteratively the master problem and the pricing problem for every $t \in[0, T]$, generating new subsets $S$ of UAVs with lower associated cost $C_{S}$, optimizing the MWCS objective involving the dual values obtained from the master problem resolution.

\section{RESUlts}

The model presented in the previous section has been implemented in Java language and solved using IBM Cplex solver 12.7.1. It has been solved on an Intel(R) Core(TM) i7-5500U CPU, $2.40 \mathrm{GHz}, 16 \mathrm{~Gb}$ RAM machine, under Microsoft 8.1 Professional operation system.

\section{A. Scenario description}

Instances are deployed in a square area of size $100 \mathrm{~m} \times$ $100 \mathrm{~m}$. We considered 10 time slots for sensor's mobility. The $2 \mathrm{D}$ coordinates of the sensors are initially chosen randomly in the monitored area. Similarly, their final location and their velocity are also chosen randomly. Then, for each time step, depending on their previous location and their velocity toward the destination, the next position is computed for every sensors. If a sensor reaches its destination coordinates before the last slot, then we assume it stays at its final location for the remaining time.

We then divide the monitored area into equal squares in which one possible point is located in the center of the square. In this way, the candidate sites for placing a flying drone form a regular grid. For each point of coordinate $\left(x_{p}, y_{p}\right)$, we set the allowed altitudes to $\{10 \mathrm{~m}, 25 \mathrm{~m}, 45 \mathrm{~m}\}$. The base station is placed at coordinates $(0.0,0.0,0.0)$. We then generated instances of size between 5 and 50 sensors, and between 75 and 300 possible locations for the UAVs. The visibility angle $\theta$ of the UAVs is set to 60 degrees, and their communication range to $30 \mathrm{~m}$.

\section{B. Performance of the model}

Table I summarizes the performance of our optimization model with subsets of covering UAVs. We present results for the tested topologies with various mobile sensors $\left|N^{t}\right|$ and 3D locations for the UAVs $|P|$.

In columns 4,6 , and 7 , we give the optimal value of the linear relaxation of the master program $\left(z_{L P}^{*}\right)$, the value of the integer master program with the set of variables of the last iteration of the column generation $\left(\bar{z}_{I L P}\right)$, and the accuracy of the integer solution $\epsilon$. When $\epsilon=0$, it means that the integer solution is optimal for our problem. This optimum is reached for different number of sensors (5 to 20, and 35) and for different number of possible positions (75 to 300). When $\epsilon>0$, its value remains very small, meaning that $\bar{z}_{I L P}$ is very close to the optimal integer value. 


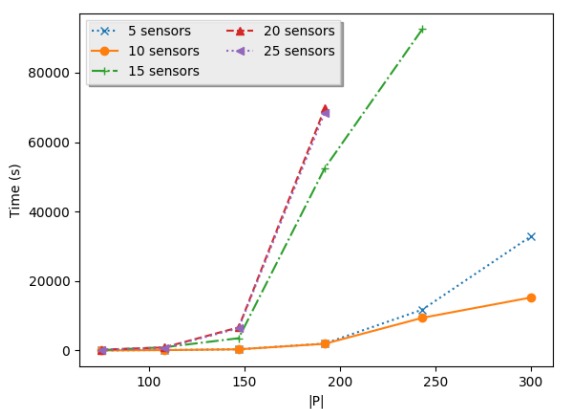

Fig. 1: Evolution of resolution time in function of the number of $3 \mathrm{D}$ positions.

The third column of the table presents the number of generated subsets of positions during the column generation process. We remark that this number is very low and always below 10 subsets. This means that the number of generated columns needed to reach the optimal value of the relaxed master problem, giving a lower bound on the optimal value of the integer program, is really small compared to the exponential size of the set of possible subsets $\mathcal{S}$. In our model, when we run the pricing program for one particular value of $t$, the new subset found actually involves adding $t$ columns in the master program, one for each time slot $t$. Consequently, a new subset of UAVs found for a specific time $t$ can be reused for other time slots in the master program. This greatly improve the column generation process. On the contrary, we must run the pricing program for each time slot $t$ in order to determine if there still exist a new subset to consider in the master program. This condition degrades the total resolution time as presented in the following.

Indeed, resolution time is presented in the fifth column of Table I. We remark that it takes less than 20 minutes to find solutions for instances with less than 147 possible 3D positions for the UAVs. Then, resolution time increases exponentially with the number of positions (see Figure 1). When the number of sensors increases, then computational time increases to several hours of resolution. This is due to the complexity of the pricing program which is $N P$-hard since it is related to a Steiner tree problem with the additional constraint that we do not know a priori the set of nodes to include into the tree. One can remark that if the higher the number of time slots, the longer the column generation process since the number of pricing resolutions will increase.

\section{Evolution of deployment among time}

In Figure 2 we investigate the number of deployed UAVs among time for different number of mobile sensors. We present the mean value among time by a bar inside each boxplot. For each topology, the number of UAVs between two consecutive time slots varies of at most 1 drone, and globally the variation of the set's size stays usually bounded by 2 . When the number of possible locations for the UAVs increases $(|P|=147)$, or when the number of sensors increases
TABLE I: Computational results of our deployment model.

\begin{tabular}{|c|c|c|c|c|c|c|}
\hline$\left|N^{t}\right|$ & $|P|$ & $\begin{array}{c}\# \\
\text { generated } \\
\text { sets }\end{array}$ & $z_{L P}^{*}$ & Time (s) & $\bar{z}_{I L P}$ & $\epsilon$ \\
\hline$\overline{5}$ & 75 & 5 & 3424.389 & 25.668 & 3424.389 & $\overline{00}$ \\
\hline 10 & - & 6 & 3905.826 & 31.473 & 3927.719 & $5.6 \times 10^{-} 3$ \\
\hline 15 & - & 8 & 5657.524 & 136.312 & 5707.063 & $8.7 \times 10^{-} 3$ \\
\hline 20 & - & 9 & 6096.653 & 249.439 & 6146.192 & $8.1 \times 10^{-} 3$ \\
\hline 25 & - & 10 & 6468.669 & 233.053 & 6484.211 & $2.4 \times 10^{-} 3$ \\
\hline 30 & - & 10 & 6574.307 & 276.054 & 6598.206 & $3.6 \times 10^{-} 3$ \\
\hline 35 & - & 9 & 7081.05 & 537.511 & 7081.05 & 0 \\
\hline 40 & - & 8 & 7184.666 & 371.639 & 7203.332 & $2.6 \times 10^{-} 3$ \\
\hline 45 & - & 10 & 7501.873 & 517.278 & 7536.078 & $4.6 \times 10^{-} 3$ \\
\hline 50 & - & 10 & 7705.093 & 650.059 & 7718.27 & $1.7 \times 10^{-} 3$ \\
\hline 5 & 108 & 6 & 3227.604 & 144.652 & 3235.296 & $2.4 \times 10^{-} 3$ \\
\hline 10 & - & 9 & 3522.636 & 120.476 & 3545.914 & $6.6 \times 10^{-} 3$ \\
\hline 15 & - & 8 & 4958.595 & 935.043 & 5046.899 & $1.8 \times 10^{-} 2$ \\
\hline 20 & - & 7 & 5183.688 & 888.504 & 5183.688 & 0 \\
\hline 25 & - & 8 & 5312.243 & 745.25 & 5330.563 & $3.4 \times 10^{-} 3$ \\
\hline 30 & - & 9 & 5345.651 & 769.817 & 5403.246 & $1.1 \times 10^{-} 2$ \\
\hline 35 & - & 8 & 5885.56 & 1315.132 & 5917.416 & $5.4 \times 10^{-} 3$ \\
\hline 40 & - & 10 & 6106.151 & 1150.683 & 6188.343 & $1.3 \times 10^{-} 2$ \\
\hline 45 & - & 10 & 6360.619 & 911.651 & 6376.655 & $2.5 \times 10^{-} 3$ \\
\hline 50 & - & 10 & 6559.157 & 1303.261 & 6588.797 & $4.5 \times 10^{-} 3$ \\
\hline 5 & 147 & 6 & 2889.468 & 365.313 & 2889.468 & 0 \\
\hline 10 & - & 7 & 3128.349 & 351.032 & 3128.349 & 0 \\
\hline 15 & - & 8 & 4606.988 & 3553.59 & 4606.988 & 0 \\
\hline 20 & - & 8 & 4769.846 & 6670.563 & 4774.719 & $1.0 \times 10^{-} 3$ \\
\hline 25 & - & 9 & 5031.934 & 6415.417 & 5047.334 & $3.1 \times 10^{-} 3$ \\
\hline 30 & - & 9 & 5088.588 & 6440.275 & 5092.94 & $8.6 \times 10^{-} 4$ \\
\hline 35 & - & 8 & 5472.193 & 8352.025 & 5517.763 & $8.3 \times 10^{-} 3$ \\
\hline 40 & - & 9 & 5638.079 & 7682.315 & 5674.783 & $6.5 \times 10^{-} 3$ \\
\hline 45 & - & 10 & 5899.808 & 11361.689 & 5922.879 & $3.9 \times 10^{-} 3$ \\
\hline 50 & - & 9 & 5973.393 & 8723.237 & 5996.662 & $3.9 \times 10^{-} 3$ \\
\hline 5 & 192 & 6 & 2782.009 & 2000.798 & 2782.009 & 0 \\
\hline 10 & - & 8 & 3174.343 & 1938.628 & 3184.558 & $3.2 \times 10^{-} 3$ \\
\hline 15 & - & 10 & 4774.253 & 52511.092 & 4789.456 & $3.2 \times 10^{-} 3$ \\
\hline 20 & - & 10 & 4879.655 & 69763.808 & 4906.987 & $5.6 \times 10^{-} 3$ \\
\hline 25 & - & 10 & 5077.339 & 68535.967 & 5095.287 & $3.5 \times 10^{-} 3$ \\
\hline 5 & 243 & 6 & 2652.332 & 11764.966 & 2690.736 & $1.4 \times 10^{-} 2$ \\
\hline 10 & - & 8 & 3001.447 & 9415.008 & 3010.627 & $3.0 \times 10^{-} 3$ \\
\hline 15 & & 8 & 4429.92 & 92514.845 & 4442.771 & $2.9 \times 10^{-} 3$ \\
\hline 5 & 300 & 8 & 2576.889 & 32964.699 & 2576.889 & 0 \\
\hline 10 & & 8 & 2761.192 & 15286.829 & 2776.259 & $5.4 \times 10^{-} 3$ \\
\hline
\end{tabular}

$\left(\left|N^{t}\right|=45\right.$ or 50 for $|P|=75$ ), then the number of UAVs deployed at each time remains constant. Then, we can see that the number of UAVs deployed to cover the targets increases with the number of sensors to cover. Since we optimize the sum of the deployment cost of the selected sets among time, then the number of deployed UAVs at each time slot remains minimum to cover the target. However, we can remark that the number of UAVs is less than or equal to the number of sensors. Indeed, we do not restrict an UAV to cover more than one sensor, optimizing the deployment cost in an effective way. Finally, in Figure 3, we depict the evolution of the cost of the selected sets among time. We seek to keep the cost as constant as possible due to the second part of the objective function of our optimization problem. However the sensors mobility enforces the UAVs to often change positions. In particular, when the number of sensors is important, then a configuration when several sensors are located close to each other can change drastically the cost of the subsets. For instance, the case for $\left|N^{t}\right|=40$ and $|P|=108$ has a drop of the cost at time $t=1$. This is due to the removal of an UAV located far away from the base station. The UAV is then needed again, 


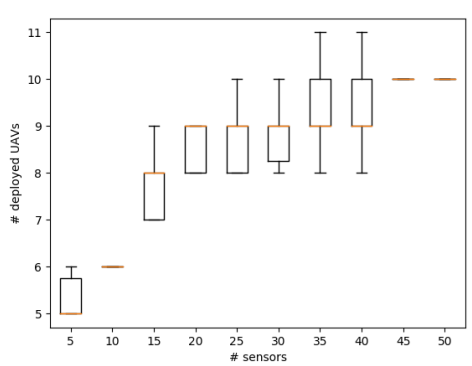

(a) $|P|=75$.

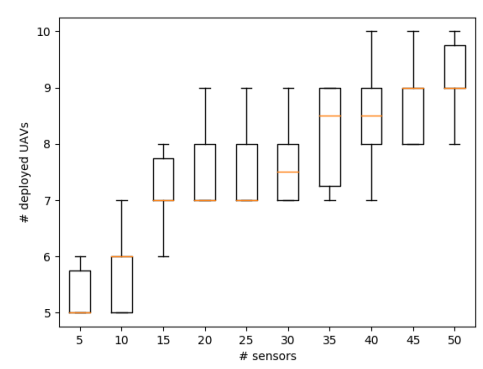

(b) $|P|=108$.

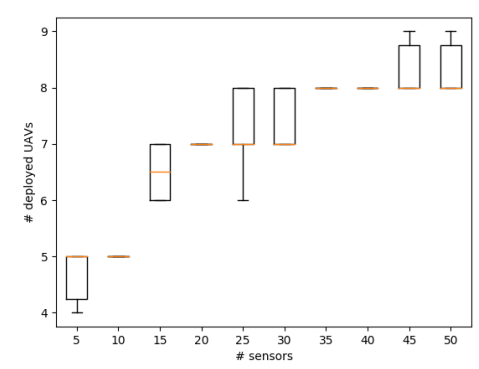

(c) $|P|=147$.

Fig. 2: Distribution of the size of selected subsets of UAV positions among time.

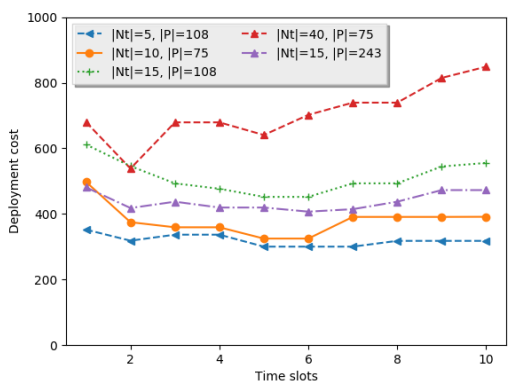

Fig. 3: Evolution of deployment cost among time.

making the cost become higher again. Moreover, the evolution of cost is optimized only for consecutive time slots. Due to the mobility pattern of the sensors, small changes are needed at each time, either by adjusting the altitude of the UAVs, or by changing the location of the most remote UAVs.

\section{CONCLUSIONS}

In this paper, we look at the mobile sensor coverage problem with UAVs and propose a new decomposition model to solve it using column generation. We show that our model can solve exactly the coverage problem while ensuring that the UAVs form a connected backbone to collect information from the mobile sensors to a central base station. Our model provides solutions with minimum deployment cost, minimizing jointly the number of deployed UAVs, their 3D distance with the base station, and the changes between consecutive time slots. It also show that only a very small number of connected sets of UAVs are sufficient to continuously monitor the mobile sensors among time. Future work will then be dedicated to find approximation algorithms to solve the pricing program efficiently and obtain solutions for larger sets of 3D locations, and to quantify the trade-off between keeping the same set of UAVs as long as possible among time and changing slightly the position of some UAV at each time to follow the sensors.

\section{ACKNOWLEDGMENT}

This work is supported by ANR program "Investments for the Future" under reference ANR-11-LABX-0031-01.

\section{REFERENCES}

[1] J. Enright, E. Frazzoli, M. Pavone, and K. Savla, "UAV routing and coordination in stochastic and dynamic environments," in Handbook of Unmanned Aerial Vehicles. Springer, 2014.

[2] S. Hayat, E. Yanmaz, and R. Muzaffar, "Survey on unmanned aerial vehicle networks for civil applications: A communications viewpoint," IEEE Communications Surveys Tutorials, vol. 18, no. 4, pp. 2624-2661, Fourthquarter 2016.

[3] C. Cambra, S. Sendra, J. Lloret, and L. Parra, "Ad hoc network for emergency rescue system based on unmanned aerial vehicles," Network Protocols and Algorithms, vol. 7, no. 4, pp. 72-89, Dec 2015.

[4] D. Orfanus, E. P. de Freitas, and F. Eliassen, "Self-organization as a supporting paradigm for military uav relay networks," IEEE Communications Letters, vol. 20, no. 4, pp. 804-807, April 2016.

[5] M. Mozaffari, W. Saad, M. Bennis, and M. Debbah, "Efficient deployment of multiple unmanned aerial vehicles for optimal wireless coverage," IEEE Communications Letters, vol. 20, no. 8, pp. 1647-1650, Aug 2016.

[6] A. Ahmadzadeh, G. Buchman, P. Cheng, A. Jadbabaie, J. Keller V. Kumar, and G. Pappas, "Cooperative control of uavs for search and coverage," in AUVSI Unmanned Systems Conference, 2006.

[7] E. Yanmaz, "Connectivity versus area coverage in unmanned aerial vehicle networks," in 2012 IEEE International Conference on Coтmunications (ICC), June 2012, pp. 719-723.

[8] M. M. Zavlanos, M. B. Egerstedt, and G. J. Pappas, "Graph-theoretic connectivity control of mobile robot networks," Proceedings of the IEEE, vol. 99, no. 9, pp. 1525-1540, Sept 2011.

[9] Z. Becvar, M. Vondra, P. Mach, J. Plachy, and D. Gesbert, "Performance of mobile networks with uavs: Can flying base stations substitute ultradense small cells?" in 23th European Wireless Conference, May 2017.

[10] T. Razafindralambo, M. Erdelj, D. Zorbas, and E. Natalizio, "Spread and shrink: Point of interest discovery and coverage with mobile wireless sensors," Journal of Parallel and Distributed Computing, vol. 102, no. Supplement C, pp. 16 - 27, 2017.

[11] J. Chen and D. Gesbert, "Optimal positioning of flying relays for wireless networks: A los map approach," in 2017 IEEE International Conference on Communications (ICC), May 2017, pp. 1-6.

[12] F. Alduraibi, N. Lasla, and M. Younis, "Coverage-based node placement optimization in wireless sensor network with linear topology," in 2016 IEEE International Conference on Communications (ICC), May 2016.

[13] C. Dixon and E. W. Frew, "Optimizing cascaded chains of unmanned aircraft acting as communication relays," IEEE Journal on Selected Areas in Communications, vol. 30, no. 5, pp. 883-898, June 2012.

[14] S. Quintero, F. Papi, D. Klein, L. Chisci, and J. Hespanha, "Optimal uav coordination for target tracking using dynamic programming," in Decision and Control (CDC), 2010 49th IEEE Conference on, dec. 2010, pp. $4541-4546$.

[15] C. Caillouet and T. Razafindralambo, "Efficient deployment of connected unmanned aerial vehicles for optimal target coverage," in IEEE GIIS, Oct. 2017.

[16] D. Zorbas, L. Di Puglia Pugliese, T. Razafindralambo, and F. Guerriero, "Optimal drone placement and cost-efficient target coverage," Journal of Network and Computer Applications, vol. 75, pp. 16 - 31, 2016. 